\section{$\underset{\substack{\text { hommes } \\ \text { \& migrations }}}{ }$}

\section{Hommes \& migrations}

Revue française de référence sur les dynamiques

migratoires

$1300 \mid 2012$

Nouveaux modèles migratoires en Méditerranée

\title{
Modèles migratoires en France
}

crise économique et reconfiguration

\section{Messamah Khelifa}

\section{(2) OpenEdition}

1 Journals

\section{Édition électronique}

URL : http://journals.openedition.org/hommesmigrations/911

DOI : 10.4000/hommesmigrations.911

ISSN : 2262-3353

Éditeur

Musée national de l'histoire de l'immigration

\section{Édition imprimée}

Date de publication : 1 novembre 2012

Pagination : 6-16

ISSN : 1142-852X

\section{Référence électronique}

Messamah Khelifa, « Modèles migratoires en France », Hommes \& migrations [En ligne], 1300 | 2012,

mis en ligne le 01 novembre 2014, consulté le 01 mai 2019. URL : http://journals.openedition.org/

hommesmigrations/911; DOI : 10.4000/hommesmigrations.911 


\section{Modèles migratoires en France \\ Crise économique et reconfiguration}

Par Messamah Khelifa, maître de conférences en économie, université Paris-VIII

En ces temps de crise, l'économie mondiale se reconfigure. Les firmes internationales développent de nouvelles stratégies de captation des savoirs, accélérant la circulation des personnes à haut potentiel. Dans le même temps, les politiques restrictives des pays occidentaux en matière de migration freinent les déplacements des personnes peu qualifiées. Partagé entre la protection des emplois nationaux et son ambition de développement mondialisé, le système néolibéral peine à évaluer le potentiel économique de l'immigration. 
L'économie de la connaissance marque une inflexion du système productif qui va accélérer les mutations du modèle migratoire traditionnel. Les emplois qui déclinent le plus rapidement sont ceux que le changement technologique remet en cause : les OS du tertiaire ont remplacé ceux de l'industrie tout en reproduisant d'autres formes du taylorisme. Cet éclatement des rapports de production révèle les besoins de la nouvelle économie que la France doit nécessairement combler par des apports extérieurs "choisis". La France se trouve confrontée à la reconstruction d'un modèle migratoire "hybride". La crise du modèle organisationnel "fordien" signifie une recomposition du mode de production international, qui se traduit par une bipolarisation de la globalité migratoire. La mondialisation et les effets des nouvelles technologies de l'information et de la communication (NTIC) n'ont fait qu'accentuer la restructuration du marché international du travail durant cette dernière décennie en deux sous-ensembles inégaux.

Le premier concerne le pôle traditionnel des soutiers de l'industrialisation façonné depuis les années 1960. La forte croissance économique a entraîné des pénuries de main-d'cuvre qui se sont traduites par une forte immigration de travailleurs peu qualifiés et interchangeables sur le marché du travail. "Les immigrés ont fourni à l'industrie la main-d'euvre dont elle a besoin, constituant un indispensable élément de souplesse $e^{(1)}$." Les immigrés ont été le réservoir "taillable et corvéable" de l'industrialisation, car il s'agissait d'ouvriers non qualifiés. Ils ont été la cheville ouvrière de la valeur ajoutée durant la période des Trente Glorieuses.

Après la Seconde Guerre mondiale, ils participent à la reconstruction de l'économie et contribuent à la croissance de l'industrie française. Comme le souligne Jean-Pierre Garson, "les immigrés contribuent à la fois à l'offre de travail et à la demande de biens et services. En général l'immigration accompagne la croissance plus qu'elle ne la provoque ${ }^{(2)}$." Le choc pétrolier de 1973-1974, révélateur de la crise structurelle du capitalisme, va marquer la fin de l'âge d'or du cycle migratoire traditionnel. La nouvelle économie va rejeter progressivement toutes les valeurs-travail considérées comme inutiles par le marché du travail à cause de leur incapacité supposée à tenir leur place dans le système productif. Ainsi se constitue un pôle résiduel de travailleurs non qualifiés considérés comme les "bouche-trous" d'une industrie en pleine mutation.

\section{Crise du fordisme et dysfonctionnement du modèle migratoire "standard"}

Une partie de cette masse (femmes, mineurs, exilés politiques...), quel que soit son niveau de formation, trouve à s'employer dans les services divers : commerce, hôtelrestaurant, transport, nettoyage, aide aux personnes âgées ou aux handicapés. Des 
emplois peu attirants ou exigeants en termes de disponibilité qu'occupent parfois des diplômés asiatiques, maghrébins ou africains, et qui sont souvent mal payés. Ce pôle mineur créé par la nouvelle économie regroupe tous les laissés-pour-compte de la crise mondiale. Cette exclusion a un coût, elle n'est pas seulement la conséquence des licenciements boursiers. Elle s'explique aussi par les mutations technologiques et la redéfinition de la place du travail qui a transformé la vie de ces migrants et leurs comportements. "Plus que les oppositions idéologiques, c'est la fin du fordisme et du taylorisme qui alimente les réflexions actuelles sur les questions de justice, d'équité sociale par rapport aux différentes circulations migratoires créatrices de la richesse mondiale ${ }^{(3)}$." Ces mutations mettent en relief une nouvelle catégorie en pleine gestation qui est à la recherche de son positionnement mondial : la matière grise communément présentée comme bénéfique car conquérante à long terme.

C'est l'émergence d'un deuxième pôle de compétitivité essayant d'attirer toutes les mains-d'œuvre hautement qualifiées, pour réguler les différents segments du marché international du travail, qui va engendrer la formation mondiale d'un mercato des compétences. Cette immigration de travailleurs du savoir va constituer le nouveau gisement d'excellence créateur de valeur ajoutée pour les pays de l'Organisation de coopération et de développement économique (OCDE). Elle correspond aux besoins de la nouvelle économie de la connaissance qui met déjà en relief le retard technologique de la France en termes d'innovation, de formation, de recherche et de développement. Pour adapter son coût du travail aux évolutions de la nouvelle économie internationale, la France doit identifier et déployer des positionnements stratégiques judicieux afin de détourner une partie des compétences mondiales et, plus particulièrement, celles de ses anciennes colonies francophones. Pour cela, elle doit surtout valoriser tous les atouts de son environnement pour avoir une chance d'attirer les meilleurs cerveaux et rattraper le "gap technologique".

Ces dernières années, l'évolution des nouvelles technologies de l'information et de la communication et leur application ont changé totalement la politique migratoire qui privilégie essentiellement les flux des savoirs. Les différentes formes d'intervention des États ont contribué à façonner un "nouveau marché des talents" qui est devenu très concurrentiel mais qui fonctionne souvent sans règles ni éthique. L'automaticité des mécanismes de ce marché du travail permet de masquer les règles discriminatoires des politiques migratoires des États du Nord qui encouragent principalement l'exode des cerveaux.

La crise, les NTIC et le Printemps arabe ont donc changé la donne migratoire, mettant en relief les contradictions des politiques subies par le Sud et révélant en même temps le pillage "légal" et illégal de leur capital humain. Cette déterritorialisation des hommes, 
sous forme d'accords d'association ou de coopération scientifique, facilite surtout la mobilité des élites et leur attractivité. Cela se traduit souvent par un braconnage "déguisé" pour satisfaire tous les objectifs de Lisbonne en 2000, notamment "l'Europe de la connaissance". Pour faire face aux nouvelles formes de compétitivité internationale, l'Europe doit réinventer d'autres stratégies pour mieux maîtriser les mobilités scientifiques euro-méditerranéennes. Cette mondialisation des compétences est en quête de nouvelles règles de régulation des mouvements d'une main-d'cuvre qualifiée postfordiste.

\section{Mondialisation, crise du modèle néolibéral et reconfiguration "institutionnelle" des mobilités euro-méditerranéennes}

La crise économique et financière de cette dernière décennie, conjuguée à l'effet du Printemps arabe, révèle une plus grande complexité du phénomène migratoire actuel par rapport aux différentes crises depuis 1929, qui n'ont fait qu'accélérer l'éclatement d'un marché du travail formaté par les Trente Glorieuses. Les années 1990 vont marquer un changement d'époque par le basculement vers de nouveaux paradigmes productifs. Les besoins par rapport à l'évolution des NTIC sont plus diffus. Ils sont du même coup moins faciles à gérer pour les responsables politiques afin de mieux réguler le fonctionnement du marché du travail des savoirs.

À la fin du $\mathrm{XX}^{\mathrm{e}}$ siècle, les TIC ont rendu possible le déploiement à grande échelle des méthodes expérimentées au Japon dans les années 1960 et connues sous le terme de "toyotisme". "Elles ont pris toute leur part au développement du juste-à-temps dans l'industrie, favorisé le sur-mesure et permis, via la mise en réseau, une meilleure condition des échanges avec leurs fournisseurs et les sous-traitants. Elles ont ainsi multiplié les possibilités d'externalisation de la main-d'euvre parfois à très longue distance ${ }^{(4)}$."

Les transformations postfordistes du travail sont sans doute à l'origine du réexamen du problème des sources de la croissance qui, au sein de l'orthodoxie néoclassique, a conduit au développement des nouvelles théories dites de "la croissance endogène". Elle a depuis longtemps consacré le capital humain et la recherche et développement comme des facteurs importants de la croissance, du fait des externalités positives qu'elles sont capables de générer au sein des filiales des firmes transnationales.

Cette mutation de l'entreprise globale vers l'entreprise réseau révèle à la fois un autre type de cellule de production et de nouvelles mobilités internationales de salariés intra-firmes qui sont en train de naître. 
La diffusion horizontale du savoir dans la société, notamment au travers de la scolarisation généralisée et de la formation continue, apparaît comme un facteur capable de démultiplier la création de richesse et d'atteindre des rendements d'échelle croissants. On passe, d'un siècle à l'autre, d'une gestion taylorienne à une organisation immatérielle en systèmes de réseaux. Là réside peut-être l'une des clés de lecture du sens et des enjeux de la transition actuelle qui a conduit au capitalisme postindustriel et à de nouvelles stratégies de captation des savoirs par les firmes multinationales, qui n'ont fait qu'accentuer la déterritorialisation planétaire des élites.

Cela explique pourquoi les flux migratoires intellectuels se déclinent actuellement en termes géo-économiques Sud-Nord, Sud-Sud, mais surtout Nord-Sud et pays émergents (vers 2020). Ainsi se dessine une nouvelle circulation migratoire qui va brasser toutes les nationalités, toutes les catégories socioprofessionnelles et plus particulièrement les technico-scientifiques

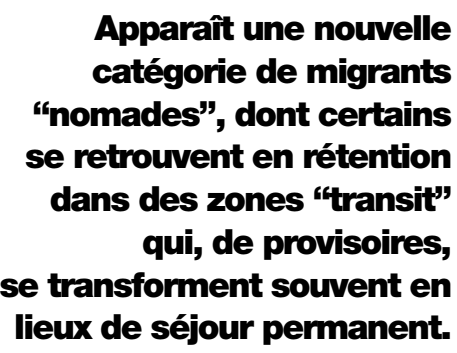
(TS); elle englobe aussi toutes les relations intergénérationnelles et interethniques qui marquent une rupture avec les vagues précédentes.

La reconfiguration de la matrice de l'allocation du capital humain révèle les caractéristiques des nouvelles mobilités euroméditerranéennes : une recomposition des flux migratoires au niveau ethnique, politique, économique, socioculturel, religieux et sécuritaire ; et, surtout, une complexité des trajectoires migratoires qui évoluent en fonction des conditions permissives d'entrée sur les différents territoires d'immigration.

La recherche des points de passage facilement accessibles va donner lieu à une autre reconfiguration territoriale des flux. Les États vont alors avoir recours à de nouveaux types de contrôle pour renforcer leur sécurité face à la clandestinité. Il en résulte une nouvelle catégorie de migrants "nomades", dont certains se retrouvent en rétention dans des zones "transit" qui, de provisoires, se transforment souvent en lieux de séjour permanent. Les migrants inventent alors de nouvelles stratégies de contournement par rapport aux différentes protections juridiques des marchés nationaux. Ils sont constamment à la recherche d'une alternative de survie.

Au niveau économique, l'apport de cette nouvelle diversité migratoire reste très contesté. Il est nécessaire d'adopter une autre démarche et de repenser les outils conceptuels qui permettent de construire une grille de lecture liée à la concurrence géo-économique imposée par la compétitivité et aux mutations géopolitiques de la circulation des compétences. 


\section{Les controverses des effets macro-économiques des coûts migratoires sur la croissance des pays développés}

De nouveaux horizons s'ouvrent pour la recherche à partir de ces nouvelles thématiques migratoires, mais en s'appuyant sur les apports des autres disciplines. La nouvelle géographie économique internationale a mis en évidence les limites restrictives des conditions micro-économiques et de micro-management des flux migratoires du modèle néolibéral. La rationalité du marché du travail et les conditions d'équilibre général de l'offre et de la demande constituent toujours le fondement de ce modèle. Dans un contexte de croissance économique, les migrations sont considérées comme un facteur de création de richesses pour les pays "d'accueil". D'un point de vue mécanique, elles ont eu un effet positif sur l'activité de croissance, comme le notait en 2006 un rapport du ministère de l'Économie. De même, certaines études et recherches d'économistes français, américains, britanniques ont abouti aux mêmes conclusions. "Aussi bien sur le plan théorique qu'empirique, les migrations hautement qualifiées ont un impact économique positif au niveau mondial. Ainsi, sous certaines conditions, les pays d'accueil ont tout à gagner à favoriser cette immigration ${ }^{(5)}$."

Les circulations migratoires deviennent le corollaire indispensable à ce processus de globalisation qui accentue la libéralisation du capital humain : lever les barrières à la mobilité des facteurs de production permet d'“égaliser" leur rémunération et d'équilibrer le marché du travail des pays développés. Les positions libérales s'accompagnent ainsi d'une volonté de décloisonner le marché du travail seulement pour l'immigration qualifiée, ce qui a souvent pour conséquence une baisse des salaires qui contribue à améliorer la compétitivité. Les théoriciens de l'organisation et du management stratégique des entreprises portent un nouveau regard sur l'allocation optimale des ressources humaines internationales, intégrant l'immigration qualifiée en tant que variable incontournable de l'ajustement structurel. Ils confirment la création de valeur ajoutée, mais la présentent souvent sous la forme d'un gain apparemment "mutuel". Ils démontrent aussi que cet apport ne peut être pérenne et qu'il est indispensable de mettre en ceuvre une politique migratoire durable.

En même temps, d'autres analyses, notamment celles du professeur d'économie de Harvard Georges Borjas, lui-même d'origine cubaine, s'opposent à toutes ces conclusions. Borjas constate plutôt une baisse des salaires des autochtones d'environ $3 \%$ en moyenne entre 1980 et 2000, via un effet de dumping, et de $9 \%$ pour les nonqualifiés avec une moindre opportunité d'emploi, surtout pour la population noire. L'analyse de cette diminution du bien-être des nationaux imputable à l'effet immigration repose sur des hypothèses erronées. 
Le choix des critères macro-économiques reste très subjectif (taille du pays, rigidité des marchés, fixation des salaires, taux d'employabilité des talents étrangers ...). Les résultats ne sont pas toujours vérifiés par les travaux empiriques. Enfin, les principales faiblesses des méthodes économétriques consistent à prédéterminer un certain nombre de variables "explicatives" pour effectuer les calculs. Les choix paraissent arbitraires et suscitent autant de débats que les résultats qu'ils engendrent.

La méthode de Paul Krugman, prix Nobel d'économie, nous semble plus intéressante. Il démontre que l'émigration latino-américaine aux USA n'a pas tant stimulé la hausse des inégalités directement par le biais du marché du travail, mais indirectement en faisant remonter sur l'échelle économique le centre de gravité du pouvoir politique qui se trouve confronté à la fois à la question du droit de vote aux étrangers, au coût de la protection sociale des populations étrangères et à toutes leurs conséquences sur les politiques budgétaires. Le problème n'est donc pas tant le nombre d'immigrés que leur répartition sur le territoire et les difficultés d'intégration auxquelles ils sont confrontés. Le droit de vote aux étrangers pourrait être un bon signal, mais cela resterait insuffisant.

\section{Revoir l'impact des migrants sur la croissance économique}

Comme le fait remarquer Élise Vincent : "Toutes ces études sont aussi souvent malmenées par d'autres interminables débats sur les 'coûts du contrôle' des flux migratoires, notamment ceux liés aux expulsions des sans-papiers et ceux consacrés à la surveillance des territoires ${ }^{(6)}$." Et comme le déplore aussi Robin Assous : "Il existe une véritable économie de la lutte contre les étrangers en séjour irrégulier, nourrie par l'augmentation de la dépense publique dans ce domaine. Le budget de l'agence européenne des frontières, Frontex, est ainsi passé de 6 millions d'euros par an à sa création en 2005 à 130 millions en 2011, auxquels s'ajoute pour la période 2007-2013 une dotation de 285 millions ${ }^{(7)}$." Claire Rodier, cofondatrice du réseau euro-africain Migreurop, soutient que les entreprises du secteur privé "sont sous-traitantes des administrations pour convoyer les migrants expulsés, fournisseurs de technologie de surveillance et de service dans des centres de rétention ${ }^{(8)}$," en même temps elle dénonce leur coût élevé et leur inefficacité. On se demande alors : à qui profite ce contrôle de l'immigration? Certaines théories mentionnent d'autres effets négatifs et s'interrogent sur les effets des récentes vagues migratoires aux États-Unis, au Canada, au Royaume-Uni, qui se traduisent parfois par une détérioration relative de la situation des primo-migrants sur le marché du travail. Cependant, la plupart des recherches qui ont été menées depuis relativisent toutes ces thèses et notamment celle 
de Borjas. L'impact calculé de l'immigration sur les salaires des locaux reste très faible. Comme le souligne Mouhoub El Mouhoud: "Au total, la très grande majorité des travaux conclut à un effet relativement modéré, plutôt négatif dans les travaux les plus anciens, et plutôt positif dans les plus récents" ${ }^{(9)}$." Il y a désormais un consensus entre les économistes sur ce point. Les études de Gianmarco Ottaviano révèlent qu'en France le marché du travail est plus rigide car plus réglementé qu'aux États-Unis : “On pourrait s'attendre à ce que les effets de l'immigration se fassent sentir sur l'emploi plutôt que sur le niveau de salaire ${ }^{(1)}$." Mais là encore les différentes recherches montrent que cet impact n'est pas significatif.

\section{La recherche de boucs émissaires}

Malgré les avancées théoriques concernant la reconnaissance du rôle collectif des connaissances, la plupart des nouvelles approches des sources de la croissance endogène continuent de se réclamer de l'héritage classique. Dès lors, la question se pose de savoir dans quelle mesure la crise économique actuelle ne peut être interprétée comme une crise du paradigme smithien ${ }^{(11)}$ se traduisant par le bouleversement de la logique de la division du travail et de la dynamique du progrès technique qui avait marqué le développement du capitalisme industriel. Les économistes ont essayé d'appréhender cette évolution en comparant seulement la place du facteur travail à travers les différents modes de production. En période de crise, le lien entre mouvement de main-d'ceuvre étrangère et accumulation du capital semble une bonne hypothèse, mais aboutit à des conclusions très controversées sur les coûts des migrations non qualifiées.

Les discours politiques n'ont fait qu'amplifier la médiatisation de la peur de l'immigration. La réaction politique a été de mettre en place différents dispositifs pour mieux la contrôler. Ils ont été imposés de façon unilatérale à chaque pays et traduisent une remise en cause du vivre ensemble. Néanmoins, cette stratégie soulève de nouveaux enjeux politico-médiatiques et idéologiques. Le durcissement des mesures protectionnistes des "valeurs républicaines" marque donc un rejet de l'étranger. On le considère comme responsable de la crise et du chômage, il est perçu comme la source d'appauvrissement de la classe ouvrière et du déclin de l'Europe.

La crise économique et sociale favorise la recherche frénétique de boucs émissaires qui conduit à banaliser les expressions du racisme au sein des sociétés européennes. Claire Rodier dénonce "limage souvent présente dans les médias et les discours politiques d'une invasion de migrants irréguliers, prédateurs et délinquants ${ }^{(12)}$ ". Ainsi se construit le dogme d'une Europe forteresse qui engendre le développement du discours anti- 
immigré suscitant la réduction de l'immigration légale du travail non qualifié. Dans la plupart des pays, les entrées sur le territoire ainsi que les listes de professions en tension sont réduites rapidement ; le critère de situation du marché du travail ("opposabilité" de l'emploi) pour autoriser ou non l'entrée sur le territoire national a été appliqué de façon très stricte, la priorité étant donnée à "la matière grise" parfois sous forme de braconnage du capital humain.

Le verrouillage des frontières signifie la fin du rêve migratoire et représente à cet égard un changement absolu de philosophie fondé sur des idées reçues : que les immigrés occupent toujours des emplois aux dépens des travailleurs nationaux, que restreindre leur nombre va favoriser la lutte contre le chômage, et qu'ils représentent un danger pour la cohésion sociale.

Mais ces mesures restrictives sont de type conjoncturel et électoraliste, elles ne peuvent constituer des réponses aux différents déséquilibres macro-économiques qui sont le fait d'un ralentissement structurel de l'activité économique. Il faut plutôt se fixer comme objectif une croissance par le haut, une croissance acquise par la compétitivité des entreprises qui doivent mettre l'accent sur la formation et la gestion éthique de leur capital humain "importé".

\section{Reconstruire un modèle migratoire durable}

La libéralisation n'a fait qu'accentuer la dislocation du marché international du travail. Elle est symbolisée par la règle des 3D (déréglementation, décloisonnement des marchés, désintermédiation financière) qui a accéléré le processus de marchandisation des savoirs du Sud, que l'on essaie de justifier logiquement à travers un discours très idéologique qui fait référence à la rationalité des lois de fonctionnement du marché. Comme le résume Pierre-Noël Giraud : "Au-delà des différences de vocabulaire, partisans du protectionnisme, du juste échange ou de la réciprocité veulent tous qu'on protège ou qu'on reconstitue quelque chose que détruisait l'échange inégal injuste ${ }^{(13)}$." Or la plupart des travaux n'apportent pas grand-chose à l'élaboration d'une politique publique. Une partie des "gains" liés à l'immigration échappent à l'économétrie, qui prend en compte plus difficilement ce qui est immatériel. Il est donc indispensable de valoriser et surtout de préserver durablement le capital humain technico-scientifique qui a été très long et très coûteux à constituer ou à attirer. Il s'agit de repenser les compétences et les professions pour lesquelles la France présentera à l'avenir un certain déficit en reconstruisant un modèle économique d'attractivité qui repose sur de nouvelles dimensions fiscalo-sociales, ces dernières pouvant aussi dissuader l'expatriation des talents. 
La construction d'une politique migratoire sélective doit prendre en compte tous ces paramètres, car il ne faut pas croire qu'il suffit d'ouvrir ses portes pour que viennent les candidats désirés, d'autant plus que la concurrence mondiale s'intensifie. Mais, en même temps, le recours à une "importation" massive de travailleurs qualifiés risque de conduire à limiter la politique d'élévation des qualifications des natifs, c'est-à-dire les investissements français dans l'enseignement supérieur.

Le déficit croissant des pays du Nord, sur les mêmes segments du marché du travail, n'a fait qu'accentuer la lutte économique entre eux. Aussi leurs regards se tournent-ils

II s'agit de repenser
les compétences et les professions
pour lesquelles la France
présentera à l’avenir un certain
déficit en reconstruisant un
modèle économique d’attractivité
qui repose sur de nouvelles
dimensions fiscalo-sociales,
ces dernières pouvant
aussi dissuader l'expatriation
des talents.
systématiquement vers le Sud. Dans ces pays, le progrès de la scolarisation et les nouveaux emplois (notamment féminins) accroissent le "capital culturel" et le "capital social" des individus qui leur permettent de partir s'installer au Nord. Pour ceux qui possèdent une qualification "élevée", le marché est devenu mondial.

La France, comme tous les "autres pays" développés, est à la recherche d'une position optimale entre "ouverture" et "restriction" des flux. Cette conception nécessite une capacité d'évaluation qualitative des futurs besoins et un calcul en termes de coûtsavantages moins inégalitaire de cet exode de cerveaux. Mais cette immigration se traduit toujours par une fuite des élites néfaste aux pays de départ, alors, doit-on limiter l'afflux des qualifiés dans le but d'éviter un "brain-drain" dans l'intérêt du pays source? Où finit l'exportation des talents et où commence la fuite des cerveaux ? Ou bien accroitre le flux des non-qualifiés dans un simple objectif humanitaire? Ou rendre service à un pays en se retenant d'attirer sa main-d'ceuvre qualifiée, mais à partir de quelle proportion de partants doit-on s'en féliciter?

Ces hypothèses, au cceur de la problématique du nouveau modèle migratoire, reflètent toutes les contradictions des politiques migratoires françaises symbolisées par les priorités politico-sécuritaires qui occultent la réalité des phénomènes migratoires. Cette dernière implique une gestion des deux volets du capital humain en termes plus éthiques pour réactiver des politiques migratoires plus équilibrées face à la crise. Pour tous ces nouveaux chantiers, il est indispensable de concevoir des outils plus efficients pour chaque objectif: en redéfinissant un pôle de gestion des valeurs-travail "inutiles" de façon solidaire, et en reconstruisant un pôle de gestion pour attirer de 
façon plus juste les talents étrangers afin de combler les écarts de compétitivité. De plus, ces migrations se sont déplacées lentement de leur espace traditionnel (le vieux continent devient très verrouillé) pour s'étendre géographiquement vers des nouveaux territoires en pleine croissance. Le nouvel enjeu Nord-Sud et pays émergents se traduit évidemment par la diffusion et l'élargissement du marché mondial des travailleurs des savoirs et des diplômés... Il est temps de repenser la vision géoéconomique imposée par l'UE aux pays du Maghreb. Le transfert de toutes les potentialités intellectuelles et de tous les gisements scientifiques nécessite une autre démarche dans un cadre partenarial durable qui associe le potentiel des savoirs entre les deux rives. Ils vont constituer un nouveau trait d'union. Il s'agit de s'interroger sur le sens de cette tendance et de mieux cerner les enjeux de la formation d'un mercato international des compétences pour affronter les défis du $\mathrm{XXI}^{\mathrm{e}}$ siècle : le technoglobalisme et le techno-terrorisme.

\section{Notes}

1. Jean-Michel Consil, "L'immigration massive des années 1920", in Alternatives économiques, $\mathrm{n}^{\circ}$ 314, juin 2012.

2. Jean-Pierre Garson. Le Monde, 23 janvier 2008.

3. Gilles Dufrenot, professeur d'économie à l'université d'Aix-Marseille.

4. Phillipe Askenaz, Les Décennies aveugles, Paris, Seuil, 2010.

5. Mark Kleinmann, Problèmes économiques, $\mathrm{n}^{\circ} 2851,2004$.

6. Le Monde, 12 avril 2011.

7. Robin Assous, "À qui profite le contrôle de l'immigration ?", in Alternatives économiques, n 56, septembre 2012.

8. Claire Rodier, Xénophobie business, les contrôles migratoires comme système, Paris, La Découverte, 2012.

9. El Mouboub Mouhoud, in Le Monde, 23 janvier 2008.

10. Gianmarco Ottaviano est chercheur à l'université Bocconi (Italie). Cité par Laurent Jeanneau,

"Pourquoi l'immigration ne menace pas l'emploi", in Alternatives économiques, n 302, mai 2011.

11. Voir Adam Smith, Recherche sur la nature et les causes de la richesse des nations, 1776.

12. Claire Rodier, op. cit.

13. Pierre-Noël Giraud, professeur d'économie à Mines-Paris Tech et à Paris-Dauphine. "Que faut-il protéger et pourquoi ?", in Alternatives économiques, hors série, n 93, 2012. 